\title{
Error Analysis and Reduction for a Simple Sensor-Microcontroller Interface
}

\author{
Angel Custodio, Ramon Pallàs-Areny, Fellow, IEEE, and Ramon Bragós, Member, IEEE
}

\begin{abstract}
Error analysis of a resistive sensor-to-microcontroller interface based on pulse-width modulation and time-ratio measurement shows that internal input and output resistances in microcontroller digital ports produce zero, gain and nonlinearity errors. The time-ratio measurement technique cancels these errors when the sensor resistance equals the reference resistor and reduces errors around that point. We propose two simple methods of reducing those errors for sensors with a wide dynamic range. Both methods use time-ratio measurements. The first method uses several reference resistors covering the sensor resistance range; the second method uses two-point calibration. The second method is more efficient and yields errors that can be smaller than $0.5 \Omega$ for a sensor resistance from about $600 \Omega$ to $3550 \Omega$.
\end{abstract}

Index Terms-Error analysis, error reduction, microcontroller interface, resistive sensor, sensor interface, time-ratio measurement.

\section{INTRODUCTION}

D IRECT sensor-to-microcontroller interfaces without intervening analog-to-digital converters (ADC) are simple to design and less expensive than interfaces based on the classical sensor-amplifier-ADC approach. Daugherty [1] described a simple analog-to-digital conversion technique based on pulsewidth modulation (PWM) using a microcontroller and Cox [2] applied it to resistive sensor interfacing (Fig. 1). In general, the time $t_{t}$ needed to charge a capacitor $C$ (initially charged at $V_{0}$ ) through a resistor $R$ to a threshold voltage $V_{t}$ from a source $V_{r}$. is

$$
t_{t}=-R C \ln \frac{V_{r}-V_{t}}{V_{r}-V_{0}}
$$

In Fig. 1, programmming a "high" level $\left(V_{\mathrm{OH}}\right)$ at output $\mathrm{O} 1$ charges $C$ through the sensor $R_{\mathrm{S}}$ up to a voltage level determined by the logic " 1 " threshold $\left(V_{\mathrm{IH}}\right)$ of input I1. Then $C$ is discharged through a protection resistor $R_{\mathrm{P}}$ by setting I1 low ("0", $\left.V_{\mathrm{OL}}\right)$. Next, $\mathrm{O} 1$ is brought to a high-impedance state by programming it as input and $\mathrm{O} 2$ is set "high" to charge $C$ through

Manuscript received May 4, 2000; revised August 28, 2001. This work was supported by the CICYT (Project TAP99-0742). A. Custodio was supported by a grant from the AECI (Spain) and by UNEXPO (Venezuela).

A. Custodio was with the Departamento Enginyeria Electrònica, Universitat Politècnica Catalunya, EPS Castelldefels, Castelldefels, Spain. He is now with the Departamento. de Electrónica, UNEXPO Puerto Ordaz, Ciudad Guayana, Venezuela. (e-mail: custodio2000@terra.es).

R. Pallàs-Areny is with the Departamento Enginyeria Electrònica, Universitat Politècnica Catalunya, EPS Castelldefels, Castelldefels, Spain (e-mail: elerpa@eel.upc.es).

R. Bragós is with the Departamento Enginyeria Electrònica, Universitat

Politècnica Catalunya, Barcelona, Spain (e-mail: rbb@eel.upc.es).

Publisher Item Identifier S 0018-9456(01)10955-1.

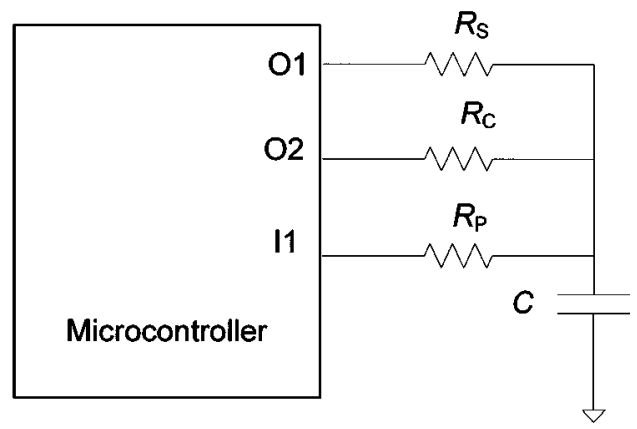

Fig. 1. Analog-to-digital conversion by pulse-width modulation (PWM).

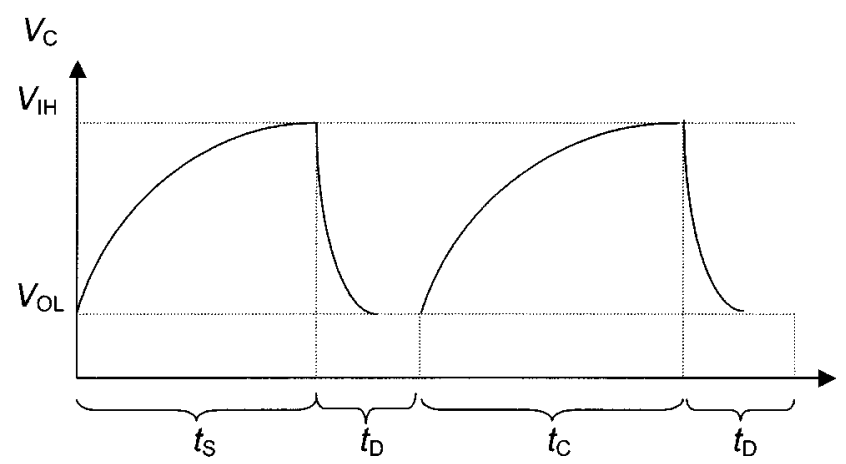

Fig. 2. Voltage waveform at port I1 (Fig. 1). $t_{\mathrm{S}}$ and $t_{\mathrm{C}}$ are the respective time intervals needed to charge $C$ through $R_{\mathrm{S}}$ and $R_{\mathrm{C}}$. The discharge time $t_{\mathrm{D}}$ through $R_{\mathrm{P}}$ is the same in both cases.

the reference resistor $R_{\mathrm{C}}$. The time taken to charge is monitored again by checking the voltage level at input I1 and $C$ is discharged again through $R_{P}$. The voltage at I1 changes between $V_{\mathrm{IH}}$ and $V_{\mathrm{OL}}$ (Fig. 2) and its pulse width depends on the time needed to charge $C$. Since $C$ is the same for both time measurements, calculating their ratio cancels the contribution of $C$ and also any possible multiplicative interference [3]. The result is

$$
R_{\mathrm{S}}^{*}=\frac{t_{\mathrm{S}}}{t_{\mathrm{C}}} R_{\mathrm{C}}
$$

where $R_{\mathrm{S}}^{*}$ is the resulting estimate of the actual sensor resistance $R_{\mathrm{S}}$.

Uncertainty in output and threshold voltages involved in the modulation process and internal microcontroller parameters such as output and input resistances and leakage currents limit the resolution and accuracy of the process depending on the range of the sensor resistance. This paper analyzes these errors and provides two simple methods of reducing them. 


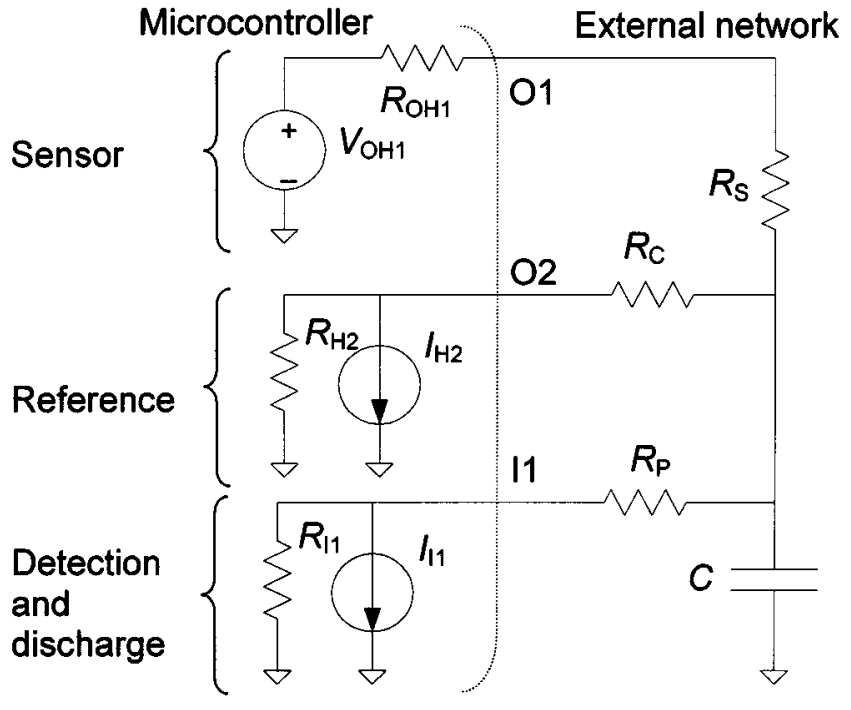

Fig. 3. Complete model for the circuit in Fig. 1 including all intervening resistances and current sources.

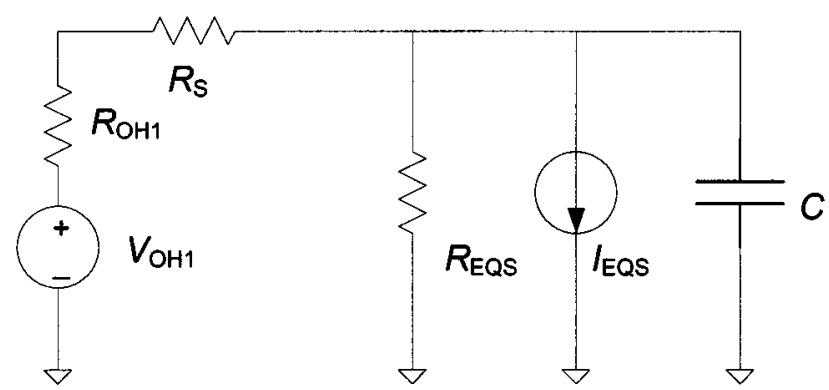

Fig. 4. Circuit equivalent to that in Fig. 3.

\section{CIRCUIT MODEL}

Fig. 3 shows the model for the circuit in Fig. 1 when $C$ charges through the sensor. The active output port has a nonzero internal resistance $R_{\mathrm{OH} 1}$. The port connected to the reference resistor $R_{\mathrm{C}}$ is set at high impedance and modeled by a finite resistance $R_{\mathrm{H} 2}$ and a leakage current $I_{\mathrm{H} 2}$. Input I1 is also modeled by a finite resistance $\left(R_{\mathrm{I} 1}\right)$ and a leakage current source $\left(I_{\mathrm{I} 1}\right)$. Ideally, $R_{\mathrm{H} 2}$ and $R_{\mathrm{I} 1}$ should be infinite and $I_{\mathrm{H} 2}$ and $I_{\mathrm{I} 1}$ should be zero. In practice, however, they are not and therefore not all the current through the sensor charges $C$. Instead, part of it flows to the other microcontroller terminals. In addition, $C$ charges not only through the sensor but also through $R_{\mathrm{C}}$ and $R_{\mathrm{P}}$.

The circuit in Fig. 3 can be simplified as shown in Fig. 4 where

$$
\begin{aligned}
R_{\mathrm{EQS}} & =\left(R_{\mathrm{I} 1}+R_{\mathrm{P}}\right) \|\left(R_{\mathrm{H} 2}+R_{\mathrm{C}}\right) \\
I_{\mathrm{EQS}} & =I_{\mathrm{I} 1} \frac{R_{\mathrm{I} 1}}{R_{\mathrm{I} 1}+R_{\mathrm{P}}}+I_{\mathrm{H} 2} \frac{R_{\mathrm{H} 2}}{R_{\mathrm{H} 2}+R_{\mathrm{C}}}
\end{aligned}
$$

When $C$ charges through $R_{\mathrm{C}}$, the model and equivalent circuit are similar to those in Figs. 3 and 4. However, in the port connected to the sensor $R_{\mathrm{S}}$ we now have $R_{\mathrm{H} 1}$ and $I_{\mathrm{H} 1}$, instead of $R_{\mathrm{H} 2}$ and $I_{\mathrm{H} 2}$ for the output port connected to the reference resistor $R_{\mathrm{C}}$. Thus, the equivalent elements in Fig. 4 are $R_{\mathrm{EQC}}$ and $I_{\mathrm{EQC}}$ obtained from (3) and (4) by replacing $R_{\mathrm{C}}$ with $R_{\mathrm{S}}$. From (1), the respective times to charge $C, t_{\mathrm{S}}$ and $t_{\mathrm{C}}$ are

$$
\begin{aligned}
& t_{\mathrm{S}}=-R_{\mathrm{EQSS}} \mathrm{Cln}\left|\frac{V_{\mathrm{S}}\left(t_{\infty}\right)-V_{\mathrm{S}}\left(t_{f}\right)}{V_{\mathrm{S}}\left(t_{\infty}\right)-V_{\mathrm{S}}\left(t_{0}\right)}\right| \\
& t_{\mathrm{C}}=-R_{\mathrm{EQCC}} \mathrm{Cln}\left|\frac{V_{\mathrm{C}}\left(t_{\infty}\right)-V_{\mathrm{C}}\left(t_{f}\right)}{V_{\mathrm{C}}\left(t_{\infty}\right)-V_{\mathrm{C}}\left(t_{0}\right)}\right|
\end{aligned}
$$

where $R_{\mathrm{EQSS}}$ and $R_{\mathrm{EQCC}}$ are the respective equivalent resistances to charge $C$ through $R_{\mathrm{S}}$ and $R_{\mathrm{C}}$, which are

$$
\begin{aligned}
R_{\mathrm{EQSS}} & =\frac{\left(R_{\mathrm{OH} 1}+R_{\mathrm{S}}\right) R_{\mathrm{EQS}}}{R_{\mathrm{OH} 1}+R_{\mathrm{S}}+R_{\mathrm{EQS}}} \\
R_{\mathrm{EQCC}} & =\frac{\left(R_{\mathrm{OH} 2}+R_{\mathrm{C}}\right) R_{\mathrm{EQC}}}{R_{\mathrm{OH} 2}+R_{\mathrm{C}}+R_{\mathrm{EQC}}} .
\end{aligned}
$$

From Fig. 2, $V_{\mathrm{S}}\left(t_{f}\right)=V_{\mathrm{C}}\left(t_{f}\right)=V_{\mathrm{IH}}$ (high-level input voltage) and $V_{\mathrm{S}}\left(t_{0}\right)=V_{\mathrm{C}}\left(t_{0}\right)=V_{\mathrm{OL}}$ (low-level output voltage). Also, from Fig. 4, the respective final voltages at the capacitor terminals are

$$
\begin{aligned}
V_{\mathrm{S}}\left(t_{\infty}\right) & =V_{\mathrm{AS}}=\frac{V_{\mathrm{OH} 1}-I_{\mathrm{EQS}}\left(R_{\mathrm{OH} 1}+R_{\mathrm{S}}\right)}{R_{\mathrm{OH} 1}+R_{\mathrm{S}}+R_{\mathrm{EQS}}} R_{\mathrm{EQS}} \\
V_{\mathrm{C}}\left(t_{\infty}\right) & =V_{\mathrm{AC}} \\
& =\frac{V_{\mathrm{OH} 2}-I_{\mathrm{EQC}}\left(R_{\mathrm{OH} 2}+R_{\mathrm{C}}\right)}{R_{\mathrm{OH} 2}+R_{\mathrm{C}}+R_{\mathrm{EQC}}} R_{\mathrm{EQC}}
\end{aligned}
$$

Because of the high value for $R_{\mathrm{H} 1}$ and $R_{\mathrm{H} 2}$ as compared to $R_{\mathrm{S}}, R_{\mathrm{C}}$ and $R_{\mathrm{P}}$, in (3) we infer $R_{\mathrm{EQS}} \approx R_{\mathrm{EQC}}$, so that when replacing (5) and (6) in (2), we can simplify this last equation to

$$
\begin{aligned}
& R_{\mathrm{S}}^{*}= \frac{t_{\mathrm{S}}}{t_{\mathrm{C}}} R_{\mathrm{C}} \\
&= \frac{R_{\mathrm{EQSS}}}{R_{\mathrm{EQCC}}} \frac{\ln \frac{V_{\mathrm{AS}}-V_{\mathrm{IH}}}{V_{\mathrm{AS}}-V_{\mathrm{OL}}}}{\ln \frac{V_{\mathrm{AC}}-V_{\mathrm{IH}}}{V_{\mathrm{AC}}-V_{\mathrm{OL}}}} R_{\mathrm{C}} \\
&= \frac{\frac{R_{\mathrm{OH} 1}+R_{\mathrm{S}}}{R_{\mathrm{OH} 1}+R_{\mathrm{S}}+R_{\mathrm{EQS}}}}{R_{\mathrm{OH} 2}+R_{\mathrm{C}}}\left(\frac{\ln A}{\ln B}\right) R_{\mathrm{C}} \\
& R_{\mathrm{OH} 2}+R_{\mathrm{C}}+R_{\mathrm{EQC}}
\end{aligned}
$$

where

$$
\begin{aligned}
& A=\frac{V_{\mathrm{AS}}-V_{\mathrm{IH}}}{V_{\mathrm{AS}}-V_{\mathrm{OL}}} \\
& B=\frac{V_{\mathrm{AC}}-V_{\mathrm{IH}}}{V_{\mathrm{AC}}-V_{\mathrm{OL}}} .
\end{aligned}
$$

Note that when $R_{\mathrm{C}}=R_{\mathrm{S}}, A \approx B$. Rearranging (11) yields

$$
R_{\mathrm{S}}^{*}=\left(R_{\mathrm{OH} 1}+R_{\mathrm{S}}\right) K\left(\frac{\ln A}{\ln B}\right) \frac{1}{\left(1+\frac{R_{\mathrm{S}}}{R_{\mathrm{OH} 1}+R_{\mathrm{EQS}}}\right)}
$$

where

$$
K=\frac{\left(R_{\mathrm{OH} 2}+R_{\mathrm{C}}+R_{\mathrm{EQC}}\right) R_{\mathrm{C}}}{\left(R_{\mathrm{OH} 2}+R_{\mathrm{C}}\right)\left(R_{\mathrm{OH} 1}+R_{\mathrm{EQS}}\right)}
$$

Finally, if $R_{\mathrm{S}}<R_{\mathrm{OH} 1}+R_{\mathrm{EQS}}$ the term after the quotient of logarithms in (14) can be expressed as a series expansion of 
$(1+z)^{-1}$ to obtain, by keeping only terms up to the second power:

$$
\begin{aligned}
R_{\mathrm{S}}^{*} \cong & \left(R_{\mathrm{OH} 1}+R_{\mathrm{S}}\right) K\left(\frac{\ln A}{\ln B}\right) \\
& \times\left(1-\frac{R_{\mathrm{S}}}{R_{\mathrm{OH} 1}+R_{\mathrm{EQS}}}+\left(\frac{R_{\mathrm{S}}}{R_{\mathrm{OH} 1}+R_{\mathrm{EQS}}}\right)^{2}\right) .
\end{aligned}
$$

This approximation yields an error smaller than 1/2 LSB for an $n$ bit digitizer (such as an analog-to-digital converter or a time counter) when

$$
1-\frac{1-\frac{R_{\mathrm{S}}}{R_{\mathrm{OH} 1}+R_{\mathrm{EQS}}}+\left(\frac{R_{\mathrm{S}}}{R_{\mathrm{OH} 1}+R_{\mathrm{EQS}}}\right)^{2}}{1+\frac{1}{R_{\mathrm{OH} 1}+R_{\mathrm{EQS}}}}<\frac{1}{2^{n+1}}
$$

which leads to

$$
R_{\mathrm{S}}<\frac{R_{\mathrm{OH} 1}+R_{\mathrm{EQS}}}{2^{(n+1) / 3}} .
$$

This condition can be less restrictive than the condition necessary to consider $R_{\mathrm{EQS}} \approx R_{\mathrm{EQC}}$ as assumed to obtain (11). Equation (14) shows that there is a zero error $\left(R_{\mathrm{OH} 1}\right)$, a gain error (because $K \ln A / \ln B \neq 1$ ) and a nonlinearity error. If ports $\mathrm{O} 1$ and $\mathrm{O} 2$ were identical, these errors would be zero for $R_{\mathrm{S}}=R_{\mathrm{C}}$. This implies selecting $R_{\mathrm{C}}=R_{0}$, the mid range value for $R_{\mathrm{S}}$. Errors for other sensor resistances depend on $R_{\mathrm{OH} 1}$, $R_{\mathrm{OH} 2}, R_{\mathrm{H} 1}, R_{\mathrm{H} 2}, V_{\mathrm{IH}}, V_{\mathrm{IL}}, V_{\mathrm{OH} 1}, V_{\mathrm{OH} 2}, I_{\mathrm{I} 1}, I_{\mathrm{H} 1}$, and $I_{\mathrm{H} 2}$. These internal microcontroller parameters depend on the technology and manufacturers do not wholly specify them. In any case, (14) shows that nonlinearity increases when $R_{\mathrm{S}}$ is close to $R_{\mathrm{OH} 1}+R_{\mathrm{EQS}}$. Since we need $R_{\mathrm{OH} 1} \ll R_{\mathrm{S}}$ to reduce the zero error, it must be $R_{\mathrm{EQS}} \gg R_{\mathrm{S}}$. The actual limits depend on the particular microcontroller and sensor used. Nevertheless, there are no limits imposed on $R_{\mathrm{S}}$ if it is close to $R_{\mathrm{C}}$. Therefore, one possible error-reduction method is to divide the sensor range into several sub-ranges and use a different reference resistor at each sub-range. This method, however, does not use the information contributed by (14).

An alternative method is to approximate (14) (or (16)) by a linear response and use two calibration resistors to determine the actual (linearized) response, in addition to the reference resistor needed for time-ratio measurement. This method is valid if the nonlinearity error does not exceed a given error target. For example, if the nonlinearity error must be less than the maximal quantization error for an $n$ bit digitizer and the condition in (18) is fulfilled, the right-most factor in (16) must not differ from 1 by more than $(1 / 2)^{n+1}$. That is

$$
\begin{aligned}
1-2^{-(n+1)} & <1-\frac{R_{0}+\left(\Delta R_{\mathrm{S}} / 2\right)}{R_{\mathrm{OH} 1}+R_{\mathrm{EQS}}}+\left(\frac{R_{0}+\left(\Delta R_{\mathrm{S}} / 2\right)}{R_{\mathrm{OH} 1}+R_{\mathrm{EQS}}}\right)^{2} \\
& <1+2^{-(n+1)}
\end{aligned}
$$

where $R_{0}-\Delta R_{\mathrm{S}} / 2<R_{\mathrm{S}}<R_{0}+\Delta R_{\mathrm{S}} / 2$. Hence, the sensor resistance variation $\left(\Delta R_{\mathrm{S}}\right)$ must fulfill the condition

$$
\left|\Delta R_{\mathrm{S}}\right|<\left(1-\sqrt{1-2^{1-n}}\right)\left(R_{\mathrm{OH} 1}+R_{\mathrm{EQS}}\right)-2 R_{0}
$$

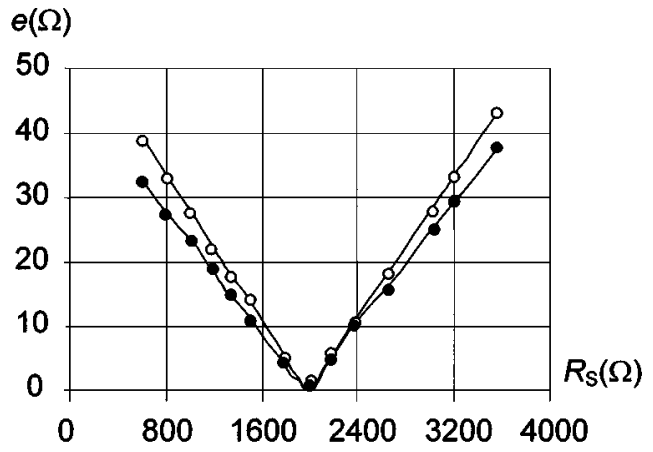

Fig. 5. Absolute error when the sensor resistance $R_{S}$ in Fig. 1 is calculated theoretically with (14) (circles) and experimentally using (2) (dots) with $R_{C}=$ $2000 \Omega$ and $618.7 \Omega<R_{\varsigma}<3553.2 \Omega$.

If $\Delta R_{\mathrm{S}}$ exceeds the limit above, or if the sensor itself is nonlinear, the measurement range can be divided into several sub-ranges and the same two-point calibration procedure applied in each sub-range.

\section{EXPERIMENTAL RESULTS}

We have built the circuit in Fig. 1 using the a PIC16C71 microcontroller and $R_{P}=220 \Omega$. Because of the inherent \pm 1 count uncertainty in time measurements $C$ must be large enough to allow sufficient time to achieve the desired resolution. We use (1) with $V_{r}=V_{\mathrm{OH}}=5 \mathrm{~V}, V_{t}=V_{\mathrm{IH}}=3.24 \mathrm{~V}$ and $V_{0}=V_{\mathrm{OL}}=0.002 \mathrm{~V}$. To obtain $2^{n}$ counts for the difference between the charging time when measuring the minimal $R_{\mathrm{S}}\left(=R_{0}-\Delta R_{\mathrm{S}} / 2\right)$ and the charging time when measuring the maximum $R_{\mathrm{S}}\left(=R_{0}+\Delta R_{\mathrm{S}} / 2\right)$ we need

$$
f_{\mathrm{e}} t_{t, \max }-f_{\mathrm{e}} t_{t, \min }=2^{n}
$$

where $f_{\mathrm{e}}$ is the equivalent frequency counted during the time interval to measure. Therefore, $C$ must fulfill the condition

$$
C>\frac{2^{n}}{f_{\mathrm{e}} \Delta R_{\mathrm{S}} \ln \frac{V_{\mathrm{OH}}-V_{\mathrm{OL}}}{V_{\mathrm{OH}}-V_{\mathrm{IH}}}} .
$$

We have measured the time interval with the internal timer of the microcontroller. The program takes three machine cycles. Since a machine cycle of the PIC16C71 takes four clock cycles $(11 \mathrm{MHz})$, we have $f_{\mathrm{e}}=(11 \mathrm{MHz}) / 12$. Hence, for $\Delta R_{\mathrm{S}}=$ $3553.2 \Omega-618.7 \Omega=2934.5 \Omega$, which is a typical range for the Pt1000 temperature sensor and, for example, $n=13$ bits, we need $C>2.9 \mu \mathrm{F}$. We have used $C=4.1 \mu \mathrm{F}$, which improves the resolution to $0.25 \Omega$. We have also used $R_{\mathrm{C}}=2000 \Omega$, that is, close to the midrange value for $R_{\mathrm{S}}$.

We measured the microcontroller parameters not specified in data sheets and calculated the coefficients in (14). Fig. 5 shows the absolute error between the estimated $R_{\mathrm{S}}^{*}$ [using (14) and experimentally using (2)] and the actual $R_{\mathrm{S}}$. The error is zero for $R_{\mathrm{S}}=R_{\mathrm{C}}$ and increases when $R_{\mathrm{S}}$ separates from $R_{\mathrm{C}}$. Using different reference resistors would keep that error below a given target. 


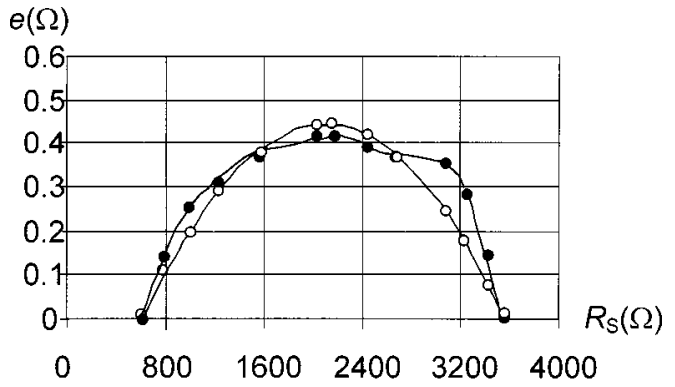

Fig. 6. Theoretical (circles) (predicted) and experimental (dots) absolute error when using time-ratio measurements and the transfer characteristic is estimated from two calibration resistors.

The least squares method shows that the best straight-line approach to (14) is

$$
R_{\mathrm{S}}^{*}=0.9667 R_{\mathrm{S}}+66.394 \Omega .
$$

Similarly, the best straight-line approach to the experimentally measured sensor resistance is

$$
R_{\mathrm{S}}^{*}=0.97 R_{\mathrm{S}}+55.83 \Omega
$$

Therefore, the experimental zero and gain errors are close to those predicted by (23). From (18), for $n=13$ bit, it should be $R_{\mathrm{S}}<300 \mathrm{k} \Omega$; hence, the approximation in (16) is acceptable. Therefore, instead of measuring the microcontroller parameters, if $\Delta R_{\mathrm{S}}$ fulfills the criterion in (20), we can use two calibration resistors to find the approximate transfer characteristic for a given microcontroller and use it to find the actual $R_{\mathrm{S}}$ from the measured $R_{\mathrm{S}}^{*}$ when in (2) we select $R_{\mathrm{C}}=R_{0}$. From (20), it should be $\Delta R_{\mathrm{S}}<3000 \Omega$, which is true in our experiment. If $R_{\mathrm{S} 2}^{*}$ and $R_{\mathrm{S} 1}^{*}$ are the estimated values for the two calibration resistors $R_{\mathrm{S} 2}$ and $R_{\mathrm{S} 1}$, the linear transfer characteristic used to find the actual $R_{\mathrm{S}}$ from the measured value $R_{\mathrm{S}}^{*}$ when $R_{\mathrm{C}}=R_{0}$ is

$$
R_{\mathrm{S}}=\frac{\left(R_{\mathrm{S}}^{*}-R_{\mathrm{S} 1}^{*}\right)\left(R_{\mathrm{S} 2}-R_{\mathrm{S} 1}\right)}{R_{\mathrm{S} 2}^{*}-R_{\mathrm{S} 1}^{*}}+R_{\mathrm{S} 1}
$$

Fig. 6 shows the predicted and experimental absolute error when applying this procedure using calibration resistors equal to the theoretical extreme for $R_{\mathrm{S}}$. The maximumal error is 0.5 $\Omega$ and depends on the available measurement resolution, which was limited to $0.25 \Omega$ for the time-interval measurement method implemented.

\section{CONCLUSION}

Sensor-to-microcontroller interfaces based on PWM and time-ratio measurement have zero, gain and nonlinearity errors due to the nonideal internal input and output resistances and leakage currents of digital ports [(14) and (16)]. Ratio measurements according to (2) reduce errors only for sensor resistances close to the reference resistor. Therefore, the first error-reduction method measures the time ratio using a reference resistor selected according to the measured sensor resistance: for a sensor with a wide resistance range, divide that range into sub-ranges and use a different reference resistors in each sub-range. However, this procedure does not use all the information provided by these reference resistors. An improved method uses a pair of calibration resistors to estimate the actual (linearized) transfer characteristic in a given sensor range and measures the time ratio using a reference resistor close to the mid range value for the sensor. This procedure, when applied to a sensor whose resistance goes ranges from about $600 \Omega$ to $3550 \Omega$, yields an absolute error of $0.5 \Omega$.

\section{ACKNOWLEDGMENT}

The authors appreciate the comments of their colleague J. Jordana.

\section{REFERENCES}

[1] K. Daugherty, Analog-to-Digital Conversion: A Practical Approach. New York: McGraw-Hill, 1994, pp. 63-93.

[2] D. Cox, "Implementing Ohmmeter/Temperature Sensor," Microchip Technology, Chandler, AZ, Appl. Note AN512, 1997.

[3] J. Huijsing, F. Riedijk, and G. Horn, "Developments in integrated smart sensors," Sens. Actuators A, vol. 43, pp. 276-288, 1994.

Angel Custodio was born in Ciudad Bolivar, Venezuela, in 1971. He received the M.Sc. degree in electronic engineering from the Polytechnic University "Antonio Jose de Sucre," Venezuela, in 1997 and the Ph.D. degree from the Universitat Politècnica de Catalunya (UPC), Barcelona, Spain, in 2001. Since 1995, he has been a member of the Department of Electronic Engineering at the Polytechnic University "Antonio José de Sucre," Ciudad Guayana, Venezuela.

Ramon Pallàs-Areny (F'98) received the Ingeniero Industrial and Doctor Ingeniero Industrial degrees from the Universitat Politècnica de Catalunya, Barcelona, Spain, in 1975 and 1982, respectively.

$\mathrm{He}$ is a Professor of electronic engineering at the same University and teaches courses in medical and electronic instrumentation. In 1989 and 1990 he was a visiting Fulbright Scholar and, in 1997 and 1998 he was an Honorary Fellow at the University of Wisconsin, Madison. In 2001 he was nominated Professor Honoris Causa by the Faculty of Electrical Engineering of the University of Cluj-Napoca, Romania. His research includes instrumentation methods and sensors based on electrical impedance measurements, sensor interfaces, ECG and arterial blood pressure measurements and electromagnetic compatibility in electronic systems. He is the author of several books on instrumentation in Spanish and Catalan, the latest one being Sensors and Interfaces, Solved Problems (Barcelona, Spain: Edicions UPC, 1999). He is also coauthor (with John G. Webster) of Sensors and Signal Conditioning, 2nd ed. (New York: Wiley, 2001) and Analog Signal Processing (New York: Wiley, 1999).

Dr. Pallàs-Areny was a recipient, with John G. Webster, of the 1991 Andrew R. Chi Prize Paper Award from the IEEE Instrumentation and Measurement Society . He is a member of the Biomedical Engineering Society and the International Society for Measurement and Control.

Ramon Bragós (M'96) received the Telecom. Eng. degree in 1991 and the Dr. Eng. degree in 1997, both from the Universitat Politècnica de Catalunya, Barcelona, Spain.

$\mathrm{He}$ is an Associate Professor at the Electronic Engineering Department of the same University and teaches courses in sensors and signal conditioning and in analog electronics. He is co-author of three books on these topics. He works in the development of automated instrumentation systems and his research is mainly focused on the development of methods and systems for the characterization of biological materials using electrical impedance spectroscopy. 